\title{
EXCHANGE OF SUBSTANCES IN AQUEOUS SOLUTION BETWEEN JOINTS AND THE VASCULAR SYSTEM ${ }^{1,2,3}$
}

\author{
By FREDERIC W. RHINELANDER, 2D, GRANVILLE A. BENNETT, ANI \\ WALTER BAUER \\ (From the Departments of Pathology and Medicine, Harvard Medical School, the Medical \\ Clinic of the Massachusetts General Hospital, and the Massachusetts Department \\ of Public Health, Boston)
}

(Received for publication June 20, 1938)

In experiments previously described (1), it was shown that materials of colloidal dimensions as the proteins of egg white and horse serum were removed from joints only by way of the lymphatics. The removal of such proteins was hastened by passive exercise of the joint.

The present series of experiments was undertaken to examine the manner of removal of materials of small molecular dimensions, present in homogeneous aqueous solutions, from normal joints, and from joints in certain induced pathological conditions. This series is part of the study to increase our knowledge of normal joint physiology and of the factors involved in the production and maintenance of joint effusions.

Solutions containing a drug having a powerful effect on the blood pressure were selected because they offered a ready and continuous means of observing their transference from joint cavity to blood stream. Preliminary experiments had shown mecholyl (acetyl-beta-methylcholine chloride, Merck), a potent and evanescent vasodepressor, relatively stable among choline compounds, to be the most suitable drug for the purposes of the experiment. Therefore mecholyl was used more extensively than any other, although adrenalin, pituitrin, and pilocarpine were also employed. One series of normal joints was studied, at rest and under passive exercise. The investigation was then extended to joints in various abnormal states.

1 This is publication Number 25 of the Robert W. Lovett Memorial for the study of crippling disease, Harvard Medical School, Boston, Massachusetts.

2 The expenses of this investigation have been defrayed by grants from the Council on Pharmacy and Chemistry of the American Medical Association, the Rockefeller Foundation, and the Commonwealth Fund.

3 Presented before the American Society for Clinical Investigation, at Atlantic City, New Jersey, May, 1936.

\section{PROCEDURE}

Knee joints of cats were used in all experiments. The cats were anesthetized intraperitoneally with nembutal, dial, or with a combination of both. The most satisfactory steady circulatory state was obtained with dial 4 $0.6 \mathrm{cc}$. per $\mathrm{kgm}$., followed in 15 minutes by nembutal 20 mgm. per kgm.

A cannula was placed in the common carotid artery for recording the blood pressure on a slowly moving smoked drum. A second cannula, bearing a short length of rubber tubing, was fixed in the axillary vein. Intravenous injections were given by syringe, with the needle inserted into this tubing, and were washed into the vein on each occasion by $1 \mathrm{cc}$. of isotonic saline delivered by pipette.

Injection of a dye into the knee joints of recently killed cats had shown that at least $1 \mathrm{cc}$. of solution could be given without escape into the structures surrounding the joint. During experimental procedures, intra-articular injections were made in $0.25 \mathrm{cc}$. of solution. Control experiments showed that mecholyl of varying strength used for such injections did not lose its potency under the conditions employed.

Exercise of a joint was carried out mechanically by flexion and extension at the rate of 60 times per minute.

At the end of each experiment, the amount of drug remaining in the joint was determined in the following manner. The joint was aspirated by means of a needle and close-fitting syringe. The few drops of synovial fluid thus obtained were placed in a small, wide test tube. The joint was then washed out with 1 cc. of normal saline, with the same syringe and without removing the needle from the joint, and the washings were added to the fluid first aspirated. This washing-out process was repeated once. The fluid first aspirated, and the joint and test-tube washings, mixed together, were then injected intravenously into the animal and the effect on the blood pressure recorded. An attempt was then made to match, by means of a known intravenous dose of mecholyl, the tracing which had thus been obtained, giving thereby an approximate estimate of the amount of drug which had been recovered from the joint. Preliminary experiments had indicated that by the above

4 A "solution of dial with urethane" supplied by the Ciba Company. 
method of washing out joints, better than 90 per cent of previously injected mecholyl could be recovered. This was deemed sufficiently accurate for the purpose of these experiments.

Accuracy of assay of the washings was sacrificed to rapidity of procedure, to assure that the cat remained in as steady a state as possible for the injection of both joints. Comparison was made of the choline-compound content of each joint. It was sufficient to establish by means of the washings that one joint contained definitely more or less residual choline-compound than the other, or that both contained approximately the same. In analyzing the results of an experiment, the point of maximal effect on each blood pressure tracing was measured, and these were compared.

No joint experiment was undertaken until repeated intravenous injections of a standard dose of mecholyl $(0.0005 \mathrm{mgm}$.) gave constant effects, thus indicating that the cat was in a steady state. This same standard intravenous dose, or one of about the same magnitude, given at the end of the experiment, served to indicate whether or not the circulatory state had altered.

A brief description of a typical experiment with the results obtained is given below.

\section{Cat Number 25}

After the usual preparation, a standard intravenous dose of mecholyl $(0.0005 \mathrm{mgm}$. $)$ was given 4 times, with an average blood pressure effect of $48 \mathrm{~mm}$. $\mathrm{Hg}$. The cat appeared to be in a steady state.

The right knee joint was injected with mecholyl $0.0025 \mathrm{mgm}$. , and kept at rest. The resulting fall of blood pressure reached a maximum of $31 \mathrm{~mm} . \mathrm{Hg}$ one minute three seconds after the injection (Figure 1). When the drug had remained in the joint for 6 minutes, the joint was washed out. Immediate intravenous injection of the joint washings produced a $50 \mathrm{~mm}$. drop of blood pressure.

The left knee joint was then injected with the same dose of mecholyl and exercised for 6 minutes. The resulting fall of blood pressure reached a maximum of $45 \mathrm{~mm} . \mathrm{Hg}, 42$ seconds after the injection. At the end of 6 minutes, the joint was washed out. The washings given at once intravenously caused a blood pressure drop of $40 \mathrm{~mm} . \mathrm{Hg}$.

Assay of washings: The initial effect of the standard intravenous dose of mecholyl had approximated the effect of the washings from the resting joint (48 and 50

NORMAL JOINTS

\section{RESTING}
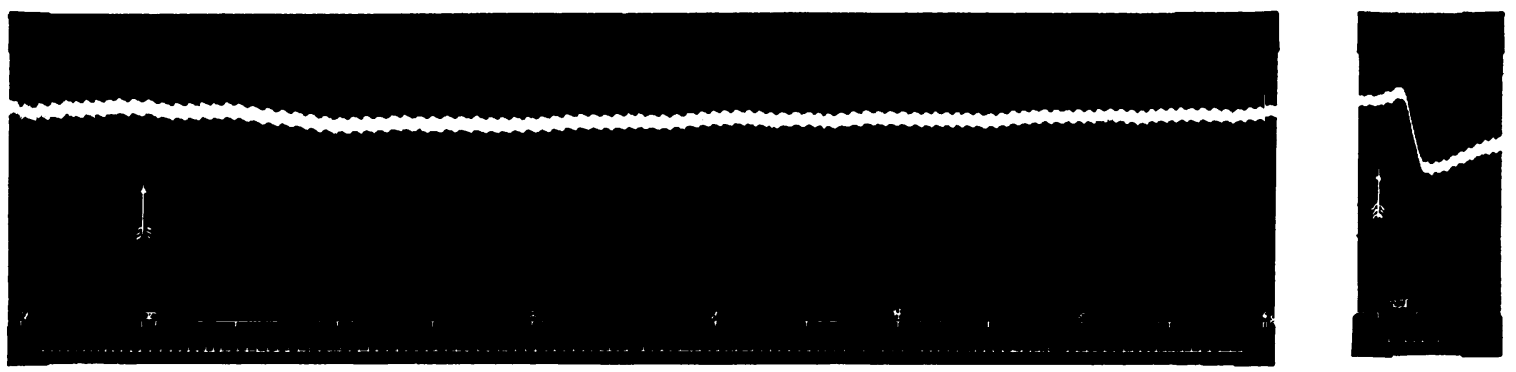

\section{EXERCISED}
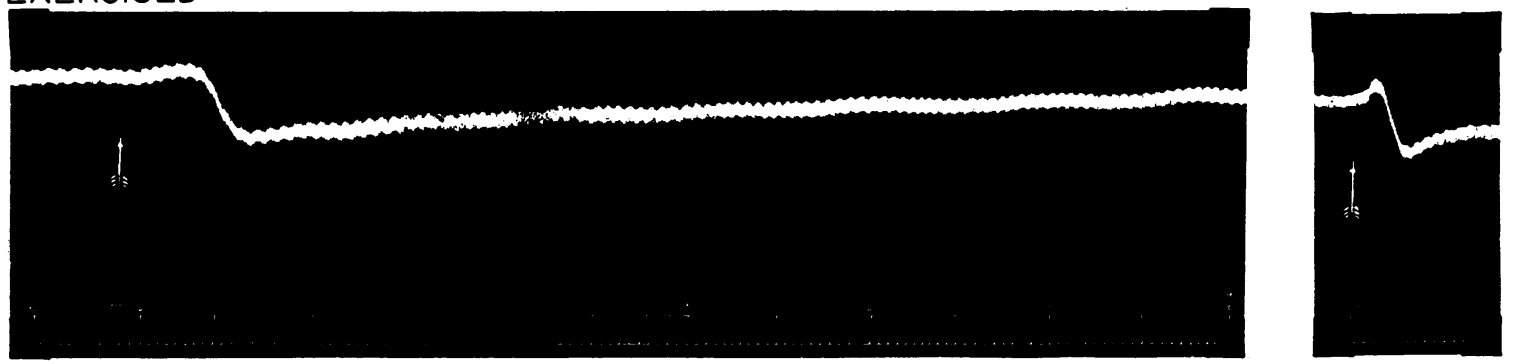

Fig. 1. Cat 25-Mecholyl (0.0025 mgm.) Injected into Each Joint

Left-hand tracings show greater blood pressure effect with exercise. Right-hand tracings show less residual mecholyl in the washings from the exercised joint.

In all figures, the tracings represent arterial blood pressure. The arrows indicate the time of intra-articular or intravenous injection. The timer records 3 -second intervals. The number of minutes after injection is also indicated in some instances.

In Figures 1, 2, and 3, the left-hand tracings show the result of the intra-articular injection of mecholyl; the right-hand tracings show the effect of the intravenous injection of the respective joint washings. 
$\mathrm{mm} . \mathrm{Hg}$, respectively), so no further assay was necessary in this case. In an attempt to approximate the effect of the washings from the exercised joint, mecholyl $0.0004 \mathrm{mgm}$. was given intravenously, leading to a blood pressure drop of $42 \mathrm{~mm}$. $\mathrm{Hg}$ (as against $40 \mathrm{~mm}$. $\mathrm{Hg}$ for the washings).

Results. There was a greater initial blood pressure effect in the experiment with the exercised joint; and the washings from this joint contained less residual mecholyl, indicating that more had been absorbed. (Compare with Table I for manner of expressing these data in brief form.)

REMOVAL OF MECHOLYL FROM NORMAL JOINTS

The removal of mecholyl from joints, normal except for the injection of the drug in $0.25 \mathrm{cc}$. of distilled water, was studied in experiments on 18 cats. Doses varying from 0.00025 to 0.01 mgm. were used.

That absorption from the joint cavity took place was shown either directly by an effect on the blood pressure of the cat soon after the injection into the joint (with the larger doses), or indirectly by failure on washing out the joint to recover the full amount of drug which had been injected (with the smaller doses). In Table I will be found the results of the experiments on normal joints.

It will be seen from this table that the variability of the effect obtained from the same dose, whether injected into a joint or a vein, bears no relation to the weights of the cats. The apparent differences in sensitivity to mecholyl of the various cats would thus appear to be one factor in the inconstant effects of the intra-articular injections, therefore no conclusions relative to small differences in permeability of the synovial membrane in different experiments can be drawn from these data.

Analysis of the complete data obtained in this group of experiments brings out the following major points of significance.

1. Mecholyl in aqueous solution was readily absorbed from all joints without significant exception.

If the absorption of mecholyl resulted in a direct effect on the blood pressure, it always did so within narrow time limits-approximately 30 to 60 seconds after injection. Absorption of the smallest doses (from 15 of 34 joints) was insufficient to produce a definite effect on the blood pressure. That this could occur, with mecholyl actually reaching the blood stream, was proved with Cat 17. The amount of mecholyl which had been absorbed from one of the joints of this cat was determined as accurately as possible by a very close assay of the washings. This amount of mecholyl was then run into the cat intravenously, by means of a constant drip apparatus, at a rate calculated to cover the same period of time that the drug had remained inside the joint. At no time during the infusion of mecholyl at this rate was any effect on the blood pressure obtained. Increasing the rate, with this and other cats, led to a slow sequence of transitory effects on the blood pressure (typical of mecholyl effects) which occurred presumably as the concentration of the drug in the blood repeatedly reached the liminal level. That, with all cats, only a single and relatively large blood pressure effect was ever obtained after intra-articular injection suggests that the rate of absorption of mecholyl from normal joints declines from the initial, rapid rate.

2. Exercise increased strikingly the rate of $a b$ sorption of mecholyl from a normal joint.

This was clearly demonstrated in 8 of the 9 experiments set up to test the point-Cats 12,14, 15, 22, 25, 26, 27, 29. (See Figure 1.) With Cats 15 and 22 this difference between the resting and the exercised joint may not be at once obvious. Although at the end of each experiment on the joints of Cat 15 equal amounts of drug had been absorbed, the drug had been longer in that joint which had not been exercised. Cat 22 was slightly more sensitive to mecholyl in the experiment with exercise, making the recorded effect of the washings greater than it should have been in comparison with the washings from the unexercised joint.

3. The longer mecholyl remained in a joint, the more of it was absorbed.

This applies only to joints in the same status regarding rest or exercise. Exercise was more potent than time in promoting absorption. However, there were three experiments in which an unusually great absorption occurred for the short time that the drug remained in the joints: both joints of Cat 12, injected with the very large dose of $0.01 \mathrm{mgm}$; and the single successfully injected joint of Cat 18, where the usual dose of $0.0025 \mathrm{mgm}$. was used. 
FREDERIC W. RHINELANDER, 2'D, GRANVILLE A. BENNETT AND WALTER BAUER

TABLE I

Condensed experimental data on the injection of mecholyl into the knee joints of normal cats

\begin{tabular}{|c|c|c|c|c|c|c|c|c|c|}
\hline \multirow{2}{*}{$\underset{\text { ner }}{\text { Cat }}$} & \multirow{2}{*}{ Weight } & \multirow{2}{*}{$\begin{array}{c}\text { Knee } \\
\text { joint }\end{array}$} & \multicolumn{2}{|c|}{$\begin{array}{c}\text { Intra-articular } \\
\text { injection }\end{array}$} & \multirow{2}{*}{$\begin{array}{c}\text { Duration } \\
\text { of } \\
\text { exercise }\end{array}$} & \multirow{2}{*}{$\begin{array}{c}\text { Time } \\
\text { drug was } \\
\text { in joint }\end{array}$} & \multirow{2}{*}{$\begin{array}{c}\text { Time } \\
\text { after } \\
\text { injection } \\
\text { of maximal } \\
\text { effect on } \\
\text { blood } \\
\text { pressure }\end{array}$} & \multirow{2}{*}{$\begin{array}{l}\text { Fall in blood } \\
\text { pressure } \\
\text { caused by } \\
\text { intravenous } \\
\text { injection } \\
\text { of joint } \\
\text { washings }\end{array}$} & \multirow{2}{*}{$\begin{array}{c}\text { Estimated } \\
\text { approximate } \\
\text { removal } \\
\text { of drug } \\
\text { from joint }\end{array}$} \\
\hline & & & Dose & $\begin{array}{c}\text { Fall in } \\
\text { blood } \\
\text { pressure }\end{array}$ & & & & & \\
\hline 28 & $\begin{array}{l}\mathrm{kgm} . \\
2.65\end{array}$ & $\begin{array}{l}\mathrm{L} \\
\mathrm{R}\end{array}$ & $\begin{array}{l}m g m . \\
0.0025 \\
0.0025\end{array}$ & \begin{tabular}{c|}
$m m . H g$ \\
24 \\
34
\end{tabular} & $\begin{array}{l}\text { None } \\
5^{\prime} 45^{\prime \prime}\end{array}$ & $\begin{array}{l}6^{\prime} \\
6^{\prime}\end{array}$ & $\begin{array}{l}48^{\prime \prime} \\
54^{\prime \prime}\end{array}$ & $\begin{array}{l}m m . \mathrm{Hg} \\
34 \\
33\end{array}$ & $\begin{array}{c}\text { per cent } \\
\text { Slightly }<80 \\
\text { Slightly }<80\end{array}$ \\
\hline 30 & 2.67 & $\underset{\mathrm{L}}{\mathrm{R}}$ & $\begin{array}{l}0.0025 \\
0.0025\end{array}$ & $\begin{array}{l}\mathbf{0} \\
\mathbf{0}\end{array}$ & $\begin{array}{r}2^{\prime} 56^{\prime \prime} \\
29^{\prime} 56^{\prime \prime}\end{array}$ & $\begin{array}{r}3^{\prime} \\
30^{\prime}\end{array}$ & . & $\begin{array}{r}36 \\
7\end{array}$ & $\begin{array}{r}\text { Slightly }>80 \\
99\end{array}$ \\
\hline 31 & 3.05 & $\begin{array}{l}\mathbf{R} \\
\mathbf{L}\end{array}$ & $\begin{array}{l}0.0025 \\
0.0025\end{array}$ & $\begin{array}{r}0 \\
16\end{array}$ & $\begin{array}{l}3^{\prime} \\
3^{\prime}\end{array}$ & $\begin{array}{l}3^{\prime} \\
3^{\prime}\end{array}$ & $1^{\prime} 3^{\prime \prime}$ & $\begin{array}{l}36 \\
40\end{array}$ & $\begin{array}{r}\text { Slightly }<80 \\
80\end{array}$ \\
\hline 12 & 3.2 & $\begin{array}{l}\mathbf{R} \\
\mathrm{L}\end{array}$ & $\begin{array}{l}0.01 \\
0.01\end{array}$ & $\begin{array}{l}30 \\
30\end{array}$ & $\begin{array}{l}2^{\prime} 13^{\prime \prime} \\
\text { None }\end{array}$ & $\begin{array}{l}3^{\prime} \\
2^{\prime} 51^{\prime \prime}\end{array}$ & $\begin{array}{l}35 \prime \prime \\
37^{\prime \prime}\end{array}$ & $\begin{array}{l}51 \\
59\end{array}$ & $\begin{array}{r}<90 \\
90\end{array}$ \\
\hline 27 & 3.31 & $\begin{array}{l}\mathrm{L} \\
\mathrm{R}\end{array}$ & $\begin{array}{l}0.0025 \\
0.0025\end{array}$ & $\begin{array}{r}0 \\
? 0\end{array}$ & $\begin{array}{l}\text { None } \\
5^{\prime} 57^{\prime \prime}\end{array}$ & $\begin{array}{l}6^{\prime} \\
6^{\prime}\end{array}$ & & $\begin{array}{l}55 \\
30\end{array}$ & $\begin{array}{r}\text { Slightly }>80 \\
\text { Much }<80\end{array}$ \\
\hline 22 & 3.6 & $\begin{array}{l}\mathrm{L} \\
\mathrm{R}\end{array}$ & $\begin{array}{l}0.0025 \\
0.0025\end{array}$ & $\begin{array}{l}26 \\
45\end{array}$ & $\begin{array}{l}\text { None } \\
3^{\prime}\end{array}$ & $\begin{array}{l}3^{\prime} \\
3^{\prime}\end{array}$ & $1^{\prime} 54^{\prime \prime}$ & $\begin{array}{l}61 \\
58\end{array}$ & $\begin{array}{l}\text { Slightly }>76 \\
\text { Slightly }>76\end{array}$ \\
\hline 11 & 3.7 & $\underset{\mathrm{L}}{\mathrm{R}}$ & $\begin{array}{l}0.01 \\
0.01\end{array}$ & $\begin{array}{l}60 \\
50\end{array}$ & $\begin{array}{l}3^{\prime} 10^{\prime \prime} \\
2^{\prime} 45^{\prime \prime}\end{array}$ & $\begin{array}{l}3^{\prime} 21^{\prime \prime} \\
2^{\prime} 54^{\prime \prime}\end{array}$ & $\begin{array}{l}42^{\prime \prime} \\
47^{\prime \prime}\end{array}$ & $\underset{64}{\text { Failed }}$ & $?$ \\
\hline 13 & 3.7 & $\begin{array}{l}\mathbf{R} \\
\mathbf{L}\end{array}$ & $\begin{array}{l}0.00025 \\
0.0005\end{array}$ & $\begin{array}{l}\mathbf{0} \\
\mathbf{0}\end{array}$ & $\begin{array}{l}1^{\prime} 32^{\prime \prime} \\
1^{\prime} 47^{\prime \prime}\end{array}$ & $\begin{array}{l}1^{\prime} 42^{\prime \prime} \\
2^{\prime} 1^{\prime \prime}\end{array}$ & & $\begin{array}{l}26 \\
46\end{array}$ & $?$ \\
\hline 14 & 3.8 & $\begin{array}{l}\mathbf{L} \\
\mathbf{R}\end{array}$ & $\begin{array}{l}0.002 \\
0.002\end{array}$ & $\begin{array}{r}40 \\
0\end{array}$ & $\begin{array}{l}3^{\prime} 3^{\prime \prime} \\
\text { None }\end{array}$ & $\begin{array}{l}3^{\prime} 36^{\prime \prime} \\
5^{\prime} 53^{\prime \prime}\end{array}$ & $51^{\prime \prime}$ & $\begin{array}{l}30 \\
46\end{array}$ & $\begin{array}{r}\text { Much }<75 \\
\text { Slightly }>75\end{array}$ \\
\hline 24 & 3.83 & $\mathbf{R}$ & 0.0025 & 12 & $1^{\prime}$ & $1^{\prime}$ & $42^{\prime \prime}$ & 51 & Slightly $<68$ \\
\hline 18 & 4.0 & L & 0.0025 & 23 & $1^{\prime} 33^{\prime \prime}$ & $2^{\prime} 45^{\prime \prime}$ & $36^{\prime \prime}$ & 46 & Slightly $<88$ \\
\hline 19 & 4.06 & $\begin{array}{l}\mathrm{R} \\
\mathrm{L}\end{array}$ & $\begin{array}{l}0.0025 \\
0.0025\end{array}$ & $\begin{array}{l}10 \\
12\end{array}$ & $\begin{array}{l}6^{\prime} \\
9^{\prime} \\
9^{\prime \prime}\end{array}$ & $\begin{array}{l}7^{\prime} 27^{\prime \prime} \\
9^{\prime} 30^{\prime \prime}\end{array}$ & $\begin{array}{l}33^{\prime \prime} \\
33^{\prime \prime}\end{array}$ & $\begin{array}{l}19 \\
18\end{array}$ & $\begin{array}{r}92 \\
\text { Slightly }>96\end{array}$ \\
\hline 15 & 4.1 & $\begin{array}{l}\mathrm{R} \\
\mathrm{L}\end{array}$ & $\begin{array}{l}0.002 \\
0.002\end{array}$ & $\begin{array}{r}20 \\
0\end{array}$ & $\begin{array}{l}2^{\prime} 30^{\prime \prime} \\
\text { None }\end{array}$ & $\begin{array}{l}3^{\prime} 38^{\prime \prime} \\
4^{\prime} 54^{\prime \prime}\end{array}$ & $1^{\prime} 7^{\prime \prime}$ & $\begin{array}{l}28 \\
28\end{array}$ & $\begin{array}{l}\text { Slightly }<75 \\
\text { Slightly }<75\end{array}$ \\
\hline 25 & 4.1 & $\begin{array}{l}\mathbf{R} \\
\mathbf{L}\end{array}$ & $\begin{array}{l}0.0025 \\
0.0025\end{array}$ & $\begin{array}{l}31 \\
45\end{array}$ & $\begin{array}{l}\text { None } \\
6^{\prime}\end{array}$ & $\begin{array}{l}6^{\prime} \\
6^{\prime}\end{array}$ & $\begin{array}{l}1^{\prime} 3^{\prime \prime} \\
42^{\prime \prime}\end{array}$ & $\begin{array}{l}50 \\
40\end{array}$ & $\begin{array}{r}\text { Slightly }>80 \\
84\end{array}$ \\
\hline 29 & 4.12 & $\stackrel{\mathrm{L}}{\mathrm{R}}$ & $\begin{array}{l}0.0025 \\
0.0025\end{array}$ & $\begin{array}{l}0 \\
0\end{array}$ & $\begin{array}{l}\text { None } \\
60^{\prime}\end{array}$ & $\begin{array}{l}60^{\prime} \\
60^{\prime}\end{array}$ & & $\begin{array}{l}24 \\
? 8\end{array}$ & $\begin{array}{r}\text { Slightly }>96 \\
\text { (?) } 100\end{array}$ \\
\hline 17 & 4.2 & $\begin{array}{l}\mathrm{R} \\
\mathrm{L}\end{array}$ & $\begin{array}{l}0.002 \\
0.002\end{array}$ & $\begin{array}{r}? 6 \\
0\end{array}$ & $\begin{array}{l}2^{\prime} 32^{\prime \prime} \\
2^{\prime} 6^{\prime \prime}\end{array}$ & $\begin{array}{l}3^{\prime} 6^{\prime \prime} \\
2^{\prime} 37^{\prime \prime}\end{array}$ & $? 44^{\prime \prime}$ & $\begin{array}{l}46 \\
52\end{array}$ & $\begin{array}{l}80 \\
80\end{array}$ \\
\hline 10 & 4.4 & $\begin{array}{l}\mathrm{R} \\
\mathrm{L}\end{array}$ & $\begin{array}{l}0.001 \\
0.01\end{array}$ & $\begin{array}{r}0 \\
25\end{array}$ & $\begin{array}{l}2^{\prime} 42^{\prime \prime} \\
\text { None }\end{array}$ & $\begin{array}{l}3^{\prime} 8^{\prime \prime} \\
3^{\prime} 18^{\prime \prime}\end{array}$ & $51^{\prime \prime}$ & $\begin{array}{l}38 \\
\text { Failed }\end{array}$ & $?$ \\
\hline 26 & 4.45 & $\begin{array}{l}\mathbf{L} \\
\mathbf{R}\end{array}$ & $\begin{array}{l}0.0025 \\
0.0025\end{array}$ & $\begin{array}{l}\mathbf{0} \\
\mathbf{0}\end{array}$ & $\begin{array}{l}\text { None } \\
8^{\prime} 57^{\prime \prime}\end{array}$ & $\begin{array}{l}9^{\prime} \\
9^{\prime}\end{array}$ & & $\begin{array}{l}40 \\
22\end{array}$ & $\begin{array}{l}\text { Slightly }>80 \\
\text { Slightly }<88\end{array}$ \\
\hline
\end{tabular}

REMOVAL OF MECHOLYL FROM ABNORMAL JOINTS

Having established the characteristics of the absorption of mecholyl from normal resting and exercised joints, a comparison was sought with joints in varying induced pathological states of an acute nature.
A. Removal of synovial fluid by saline washing

In order to determine the effect of removing synovial fluid, one or both knee joints of 15 cats were washed out twice with $1 \mathrm{cc}$. of sterile normal saline, under ether anesthesia. After each washing, the joint was aspirated as completely as 
TABLE II *

Synovial fluid removed from joints 24 hours previously by saline washing

\begin{tabular}{|c|c|c|c|c|c|c|c|c|c|c|c|c|c|c|}
\hline \multicolumn{5}{|c|}{$\begin{array}{c}\text { One joint washed out with saline; } \\
\text { both joints rested }\end{array}$} & \multicolumn{5}{|c|}{$\begin{array}{l}\text { One joint washed out with saline; } \\
\text { both joints exercised }\end{array}$} & \multicolumn{5}{|c|}{$\begin{array}{l}\text { Both joints washed out with saline; } \\
\text { one joint exercised }\end{array}$} \\
\hline \multirow{2}{*}{$\underset{\text { ber }}{\text { Cat }}$} & \multicolumn{2}{|c|}{$\begin{array}{l}\text { Greater blood } \\
\text { pressure effect }\end{array}$} & \multicolumn{2}{|c|}{$\begin{array}{c}\text { Greater } \\
\text { absorption }\end{array}$} & \multirow{2}{*}{$\underset{\text { ber }}{\text { Cat }}$} & \multicolumn{2}{|c|}{$\begin{array}{l}\text { Greater blood } \\
\text { pressure effect }\end{array}$} & \multicolumn{2}{|c|}{$\begin{array}{l}\text { Greater } \\
\text { absorption }\end{array}$} & \multirow{2}{*}{$\underset{\text { ber }}{\text { Cat }}$} & \multicolumn{2}{|c|}{$\begin{array}{l}\text { Greater blood } \\
\text { pressure effect }\end{array}$} & \multicolumn{2}{|c|}{$\begin{array}{l}\text { Greater } \\
\text { absorption }\end{array}$} \\
\hline & $\begin{array}{c}\text { Nor- } \\
\text { mal } \\
\text { joint }\end{array}$ & $\underset{\substack{\text { normal } \\
\text { joint }}}{\text { Ab- }}$ & $\begin{array}{c}\text { Nor- } \\
\text { mal } \\
\text { joint }\end{array}$ & $\underset{\substack{\text { normal } \\
\text { joint }}}{\text { Ab- }}$ & & $\begin{array}{c}\text { Nor- } \\
\text { mal } \\
\text { joint }\end{array}$ & $\underset{\substack{\text { normal } \\
\text { joint }}}{\text { Ab- }}$ & $\begin{array}{c}\text { Nor- } \\
\text { mal } \\
\text { joint }\end{array}$ & $\underset{\substack{\text { normal } \\
\text { joint }}}{\text { Ab- }}$ & & $\begin{array}{c}\text { Rest- } \\
\text { ing } \\
\text { joint }\end{array}$ & $\begin{array}{l}\text { Exer- } \\
\text { cised } \\
\text { joint }\end{array}$ & $\begin{array}{l}\text { Rest- } \\
\text { ing } \\
\text { joint }\end{array}$ & $\begin{array}{l}\text { Exer- } \\
\text { cised } \\
\text { joint }\end{array}$ \\
\hline $\begin{array}{l}\mathbf{3 7} \\
\mathbf{3 8} \\
\mathbf{3 9} \\
\mathbf{4 0} \\
\mathbf{4 1} \\
\mathbf{5 3} \\
\mathbf{5 4} \\
\mathbf{5 5}\end{array}$ & $\begin{array}{r}+t+ \\
++t \\
= \\
=\end{array}$ & $\begin{array}{r}++ \\
+++ \\
= \\
+++ \\
=\end{array}$ & $\begin{array}{r}+++ \\
x \\
++ \\
= \\
=\end{array}$ & $\begin{array}{r}x \\
+t \\
++ \\
= \\
++ \\
=\end{array}$ & $\begin{array}{l}43 \\
52 \\
72 \\
74\end{array}$ & +++ & $\begin{array}{r}+ \\
+ \\
+t-t\end{array}$ & ++ & $\begin{array}{r}t \\
+\end{array}$ & $\begin{array}{l}42 \\
50 \\
71 \\
68\end{array}$ & & $\begin{array}{r}+++ \\
++ \\
++ \\
++\end{array}$ & & $+t$ \\
\hline
\end{tabular}

* Mecholyl 0.003 mgm. was injected into each knee joint of each cat in each experiment.

The magnitude of observed difference in blood pressure effect, or in absorption, with respect to one of the two joints of a cat, is indicated by the number of + 's.

The symbol "=" indicates that the results were the same for the two joints.

The symbol " 0 " indicates that no effect occurred in this experiment.

The symbol " $x$ " indicates that this experiment was unsatisfactory.

possible. Twenty-four hours later, $0.003 \mathrm{mgm}$. of mecholyl was injected into both joints of each cat. Some of the joints were then kept at rest for 4 to 10 minutes, while others were exercised for 10 minutes. All the joints were then washed out in the usual manner and the washings assayed. Table II gives in concise form the results thus obtained.

Analysis of the data in this table shows that removal of synovial fluid 24 hours previously had no consistent effect on the absorption of mecholyl from resting joints. In some instances there appeared to be a very slight tendency for more mecholyl to be absorbed from the $j$ sint which had been washed out. When both joints were exercised, there was a tendency ( 3 of 4 cats) for more drug to be absorbed from the joint which had been washed out previously. This tendency was confirmed when the synovial fluid was washed from both joints, and then but one of the joints exercised. Here exercise appeared to enhance absorption even more than it did from the entirely normal joints previously described.

For experiments on 6 additional cats, the synovial fluid was washed out one hour, instead of 24 hours, beforehand. The results, shown in Table III, were essentially the same for exercised joints as were those obtained after the earlier

TABLE III*

Synovial fuid removed from joints one hour previously by saline washing

\begin{tabular}{|c|c|c|c|c|c|c|c|c|c|c|c|c|c|c|}
\hline \multicolumn{5}{|c|}{$\begin{array}{l}\text { One joint washed out with saline; } \\
\text { both joints rested }\end{array}$} & \multicolumn{5}{|c|}{$\begin{array}{l}\text { One joint washed out with saline; } \\
\text { both joints exercised }\end{array}$} & \multicolumn{5}{|c|}{$\begin{array}{l}\text { Both joints washed out with saline; } \\
\text { one joint exercised }\end{array}$} \\
\hline \multirow{2}{*}{$\underset{\text { ber }}{\text { Cat }}$} & \multicolumn{2}{|c|}{$\begin{array}{l}\text { Greater blood } \\
\text { pressure effect }\end{array}$} & \multicolumn{2}{|c|}{$\begin{array}{l}\text { Greater } \\
\text { absorption }\end{array}$} & \multirow{2}{*}{$\underset{\text { num- }}{\text { Cat }}$} & \multicolumn{2}{|c|}{$\begin{array}{l}\text { Greater blood } \\
\text { pressure effect }\end{array}$} & \multicolumn{2}{|c|}{$\begin{array}{l}\text { Greater } \\
\text { absorption }\end{array}$} & \multirow{2}{*}{$\underset{\text { ber }}{\text { Cat }}$} & \multicolumn{2}{|c|}{$\begin{array}{l}\text { Greater blood } \\
\text { pressure effect }\end{array}$} & \multicolumn{2}{|c|}{$\begin{array}{l}\text { Greater } \\
\text { absorption }\end{array}$} \\
\hline & 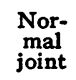 & $\underset{\substack{\text { Ab- } \\
\text { joint }}}{\stackrel{\text { joinal }}{\text { joint }}}$ & $\underset{\text { maint }}{\text { Nor- }}$ & $\begin{array}{c}\text { Ab- } \\
\text { normal } \\
\text { joint }\end{array}$ & & $\underset{\text { moint }}{\text { mor- }}$ & $\underset{\substack{\text { normal } \\
\text { joint }}}{\text { Ab- }}$ & $\underset{\text { mal }}{\text { Noint }}$ & $\underset{\substack{\text { normal } \\
\text { joint }}}{\text { ablal }}$ & & $\begin{array}{c}\text { Rested } \\
\text { joint }\end{array}$ & $\begin{array}{l}\text { Exercised } \\
\text { joint }\end{array}$ & $\begin{array}{l}\text { Rested } \\
\text { joint }\end{array}$ & $\begin{array}{l}\text { Exercised } \\
\text { joint }\end{array}$ \\
\hline $\begin{array}{l}79 \\
80\end{array}$ & $\begin{array}{l}0 \\
0\end{array}$ & $\begin{array}{l}\mathbf{0} \\
\mathbf{0}\end{array}$ & $\begin{array}{l}= \\
=\end{array}$ & $\begin{array}{l}= \\
=\end{array}$ & $\begin{array}{l}81 \\
82\end{array}$ & 0 & $\begin{array}{c}0 \\
+++\end{array}$ & & & $\begin{array}{l}76 \\
77\end{array}$ & & $\begin{array}{l}++ \\
++\end{array}$ & & $\begin{array}{l}+++t \\
+++t\end{array}$ \\
\hline
\end{tabular}

* Mecholyl $0.003 \mathrm{mgm}$. was injected into each knee joint of each cat in each experiment.

The magnitude of observed difference in blood pressure effect, or in absorption, with respect to one of the two joints of a cat, is indicated by the number of +'s.

The symbol "=" indicates that the results were the same for the two joints.

The symbol " 0 " indicates that no effect occurred in this experiment. 
washing. When both joints were rested, any possible difference between the normal and abnormal joints was too small to be detected.

In this group of experiments on washing out synovial fluid, it would be expected that depletion of synovial fluid would inhibit egress of water from the joint. But this would not prevent the rapid transference of foreign compounds (as of the drug mecholyl) from the joint cavity to the blood stream. (See experiment with pilocarpine below, where an effusion was being formed while the drug was being absorbed.) Thus the chief abnormal factor affecting the absorption of mecholyl in these experiments would appear to have been the mild irritation caused by the saline used to wash out the synovial fluid. Consequently the results in this group of experiments must be interpreted in conjunction with those of the succeeding group.

\section{B. Mild inflammation produced by saline injection}

Since previous studies with other animals had shown that injection of normal saline into joints produced a mild inflammation, with a definite rise of the synovial fluid cell count (2), this method was adopted in a series of 9 cats. One or both joints of these cats were injected with $1 \mathrm{cc}$. of sterile saline, under light ether anesthesia, and the saline was left in the joint. After 4 such injections, at intervals of 3 or 4 days, the usual mecholyl experiment was carried out on each joint, with exercise or rest for 10 minutes.

The results in this series, shown in Table IV, were essentially similar to those in the preceding series where the synovial fluid was simply washed out with saline, except that the tendency for more mecholyl to be absorbed from the abnormal joint at rest was slightly more pronounced (Figure 2).

From the above two groups of experiments, on washing out the synovial fluid with saline and on injecting saline, the following may be added to the significant observations on the removal of mecholyl from joints.

4. Mild acute inflammation led to slightly increased absorption of mecholyl from joints, more definitely with exercise than with rest.

\section{Severe inflammation produced by aleuronat injection}

Having observed previously that the injection of aleuronat ${ }^{5}$ caused marked inflammation of the synovial membrane, with fibrinous and cellular exudation (2), this substance was employed in the final series of abnormal joints. The sterile aleuronat suspension was injected, under ether anesthesia, into one or both joints of 10 cats on two occasions, one and two weeks before the joints were used for experiments identical with those of the saline-injection series. The striking results are exhibited in Table V. (See also Figure 3.)

From these results the following observation is justified.

5. Severe acute inflammation led to definitely increased absorption of an aqueous solution of mecholyl from both exercised and resting joints.

5 A suspension of 5 per cent aleuronat and 3 per cent starch in 0.5 per cent saline.

TABLE IV *

Joints mildly inflamed by injections with saline on three previous occasions

\begin{tabular}{|c|c|c|c|c|c|c|c|c|c|c|c|c|c|c|}
\hline \multicolumn{5}{|c|}{$\begin{array}{l}\text { One joint injected with saline; } \\
\text { both joints rested }\end{array}$} & \multicolumn{5}{|c|}{$\begin{array}{l}\text { One joint injected with saline; } \\
\text { both joints exercised }\end{array}$} & \multicolumn{5}{|c|}{$\begin{array}{l}\text { Both joints injected with saline; } \\
\text { one joint exercised }\end{array}$} \\
\hline \multirow{2}{*}{$\begin{array}{c}\text { Cat } \\
\text { num- } \\
\text { ber }\end{array}$} & \multicolumn{2}{|c|}{$\begin{array}{l}\text { Greater blood } \\
\text { pressure effect }\end{array}$} & \multicolumn{2}{|c|}{$\begin{array}{c}\text { Greater } \\
\text { absorption }\end{array}$} & \multirow{2}{*}{$\underset{\text { ber }}{\text { Cat }}$} & \multicolumn{2}{|c|}{$\begin{array}{l}\text { Greater blood } \\
\text { pressure effect }\end{array}$} & \multicolumn{2}{|c|}{$\begin{array}{c}\text { Greater } \\
\text { absorption }\end{array}$} & \multirow{2}{*}{$\begin{array}{c}\text { Cat } \\
\text { num- } \\
\text { ber }\end{array}$} & \multicolumn{2}{|c|}{$\begin{array}{l}\text { Greater blood } \\
\text { pressure effect }\end{array}$} & \multicolumn{2}{|c|}{$\begin{array}{c}\text { Greater } \\
\text { absorption }\end{array}$} \\
\hline & $\begin{array}{c}\text { Nor- } \\
\text { mal } \\
\text { joint }\end{array}$ & $\underset{\substack{\text { normal } \\
\text { joint }}}{\text { Ab- }}$ & $\begin{array}{c}\text { Nor- } \\
\text { mal } \\
\text { joint }\end{array}$ & $\mid \begin{array}{c}\mathrm{Ab}- \\
\text { normal } \\
\text { joint }\end{array}$ & & $\left|\begin{array}{c}\text { Nor- } \\
\text { mal } \\
\text { joint }\end{array}\right|$ & $\underset{\substack{\text { normal } \\
\text { joint }}}{\text { Ab- }}$ & $\begin{array}{l}\text { Nor- } \\
\text { mal } \\
\text { joint }\end{array}$ & $\underset{\substack{\text { normal } \\
\text { joint }}}{\mathbf{A b}-}$ & & $\begin{array}{c}\text { Rested } \\
\text { joint }\end{array}$ & $\underset{\text { joint }}{\text { Exercised }}$ & $\begin{array}{c}\text { Rested } \\
\text { joint }\end{array}$ & $\underset{\text { joint }}{\text { Exercised }}$ \\
\hline $\begin{array}{l}35 \\
45 \\
47\end{array}$ & $=$ & $\begin{array}{r}+++ \\
++ \\
=\end{array}$ & ++ & $\begin{array}{r}++ \\
=\end{array}$ & $\begin{array}{l}46 \\
65 \\
66\end{array}$ & $=$ & $\begin{array}{r}+++ \\
= \\
+\end{array}$ & & $\begin{array}{r}+++ \\
+ \\
+\end{array}$ & $\begin{array}{l}62 \\
63 \\
64\end{array}$ & $\begin{array}{l}++ \\
++\end{array}$ & $+t+t$ & & \\
\hline
\end{tabular}

* Mecholyl 0.003 mgm. was injected into each knee joint of each cat in each experiment.

The magnitude of observed difference in blood pressure effect, or in absorption, with respect to one of the two joints of a cat, is indicated by the number of +'s.

The symbol "=" indicates that the results were the same for the two joints. 
64

EXERCISED

TREATED JOINTS
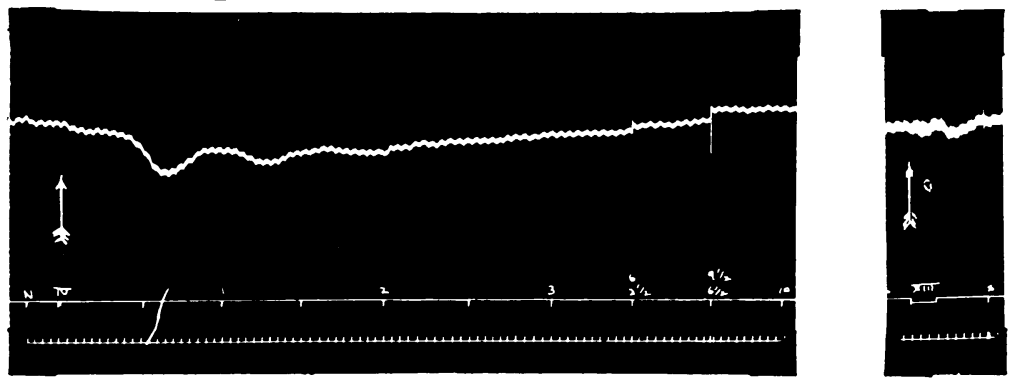

\section{RESTING}
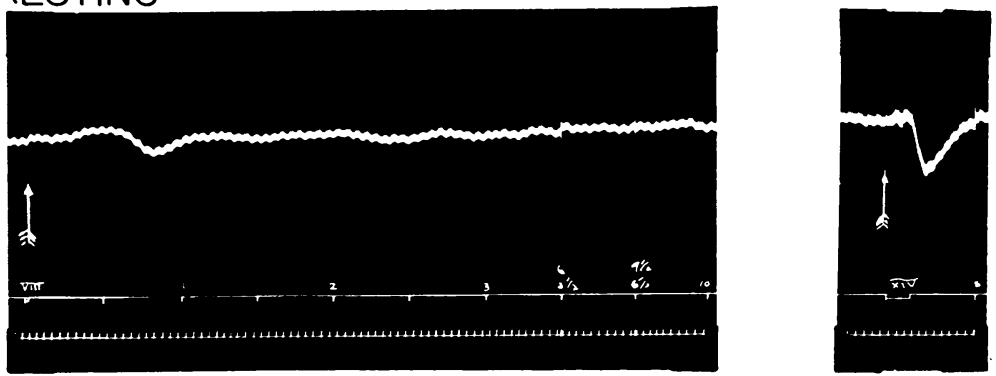

Fig. 2. Cat 64-Mecholyl (0.003 Mgar.) Injected into Each Joint

One joint rested and one exercised. Both had been injected with saline $13,10,6$, and 3 days previously. Left-hand tracings show greater immediate blood pressure effect with exercise. Right-hand tracings show less residual mecholyl in washings from exercised joint.

CONTROL DATA

The method of experiment employed in these studies suggested the necessity of the following controls, in addition to those already cited.

The propriety of using distilled water as a vehicle for introducing mecholyl into joints was checked by tests with two cats. A 0.003 mgm. dose of mecholyl dissolved in distilled water was injected into one joint, and the same dose dissolved in cat serum, into the other joint. When both joints were kept at rest, there was no blood pressure effect in either case. Assay of the wash-

TABLE $\mathrm{V}^{*}$

Joints severely inflamed by injections with aleuronat

\begin{tabular}{|c|c|c|c|c|c|c|c|c|c|c|c|c|c|c|}
\hline \multicolumn{5}{|c|}{$\begin{array}{l}\text { One joint injected with aleuronat; } \\
\text { both joints rested }\end{array}$} & \multicolumn{5}{|c|}{$\begin{array}{l}\text { One joint injected with aleuronat; } \\
\text { both joints exercised }\end{array}$} & \multicolumn{5}{|c|}{$\begin{array}{c}\text { Both joints injected with aleuronat; } \\
\text { one joint exercised }\end{array}$} \\
\hline \multirow{2}{*}{$\underset{\text { ber }}{\text { Cat }}$} & \multicolumn{2}{|c|}{$\begin{array}{l}\text { Greater blood } \\
\text { pressure effect }\end{array}$} & \multicolumn{2}{|c|}{$\begin{array}{l}\text { Greater } \\
\text { absorption }\end{array}$} & \multirow{2}{*}{$\begin{array}{c}\text { Cat } \\
\text { num- } \\
\text { ber }\end{array}$} & \multicolumn{2}{|c|}{$\begin{array}{l}\text { Greater blood } \\
\text { pressure effect }\end{array}$} & \multicolumn{2}{|c|}{$\begin{array}{l}\text { Greater } \\
\text { absorption }\end{array}$} & \multirow{2}{*}{$\begin{array}{c}\text { Cat } \\
\text { num- } \\
\text { ber }\end{array}$} & \multicolumn{2}{|c|}{$\begin{array}{l}\text { Greater blood } \\
\text { pressure effect }\end{array}$} & \multicolumn{2}{|c|}{$\begin{array}{l}\text { Greater } \\
\text { absorption }\end{array}$} \\
\hline & $\begin{array}{c}\text { Nor- } \\
\text { mal } \\
\text { joint }\end{array}$ & $\begin{array}{c}\mathrm{Ab}- \\
\text { normal } \\
\text { joint }\end{array}$ & $\begin{array}{c}\text { Nor- } \\
\text { mal } \\
\text { joint }\end{array}$ & $\begin{array}{l}\text { Ab- } \\
\text { normal } \\
\text { joint }\end{array}$ & & $\begin{array}{c}\text { Nor- } \\
\text { mal } \\
\text { joint }\end{array}$ & $\begin{array}{c}\mathrm{Ab}- \\
\text { normal } \\
\text { joint }\end{array}$ & $\begin{array}{c}\text { Nor- } \\
\text { mal } \\
\text { joint }\end{array}$ & $\begin{array}{l}\mathrm{Ab}- \\
\text { normal } \\
\text { joint }\end{array}$ & & $\begin{array}{c}\text { Rested } \\
\text { joint }\end{array}$ & $\begin{array}{l}\text { Exercised } \\
\text { joint }\end{array}$ & $\begin{array}{c}\text { Rested } \\
\text { joint }\end{array}$ & $\begin{array}{l}\text { Exercised } \\
\text { joint }\end{array}$ \\
\hline $\begin{array}{l}36 \\
44 \\
56 \\
61\end{array}$ & $\begin{array}{l}? \\
0\end{array}$ & $\begin{array}{r}+t \\
++++\frac{?}{0}\end{array}$ & $=$ & $\begin{array}{r}+++ \\
++ \\
+++ \\
=\end{array}$ & $\begin{array}{l}48 \\
49 \\
57\end{array}$ & & $\begin{array}{r}+++ \\
++++ \\
+++\end{array}$ & & $\begin{array}{r}t+t+ \\
+ \\
+t+t\end{array}$ & $\begin{array}{l}58 \\
59 \\
60\end{array}$ & & $\begin{array}{r}++++ \\
++++ \\
+++\end{array}$ & & $\begin{array}{l}++t+ \\
++t+ \\
++t+\end{array}$ \\
\hline
\end{tabular}

* Mecholyl $0.003 \mathrm{mgm}$. was injected into each knee joint of each cat in each experiment.

The magnitude of observed difference in blood pressure effect, or in absorption, with respect to one of the two joints of a cat, is indicated by the number of +'s.

The symbol "=" indicates that the results were the same for the two joints.

The symbol " 0 " indicates that no effect occurred in this experiment. 


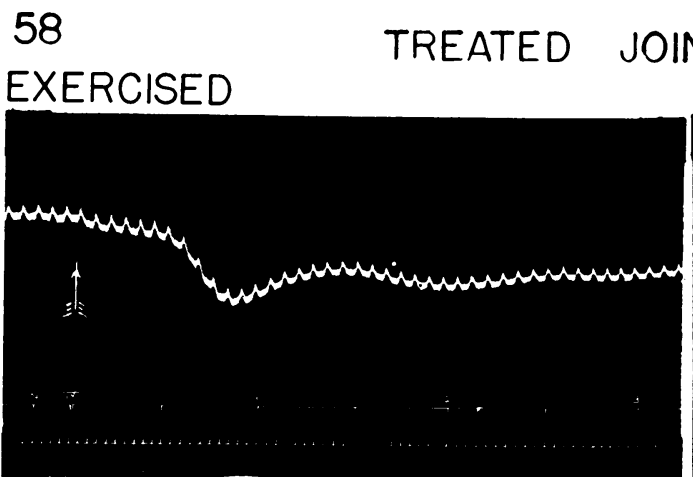

\section{RESTING}
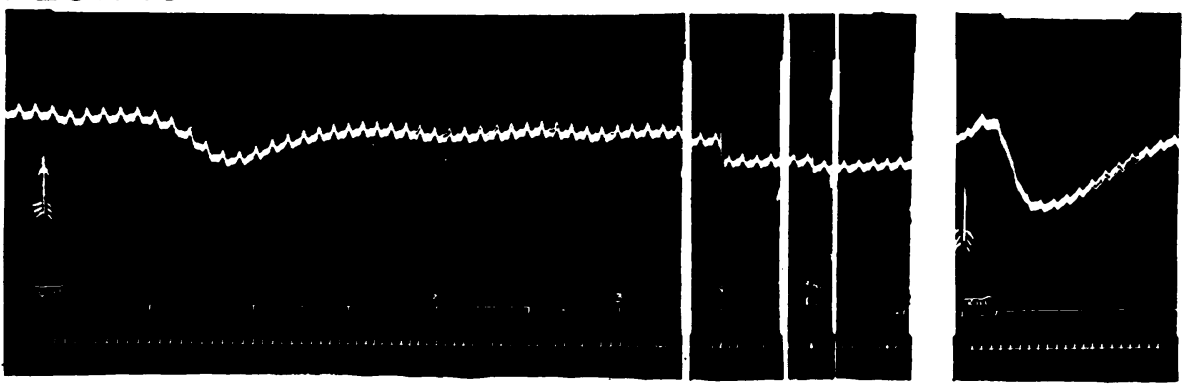

Fig. 3. Cat 58-Mfacholyi (0.003 igm.) Injected into Each Joint

One joint rested and one exercised. Both had been injected with aleuronat 11 and 6 days previously. Left-hand tracings show greater immediate blood pressure effect with exercise. Right-hand tracings show less residual mecholyl in washings from exercised joint.

ings showed equal removal of mecholyl from each joint after 10 minutes. The same experiment, but with exercise of both joints for 10 minutes, gave a somewhat greater initial blood pressure effect when serum was used. but a slightly greater total removal when mecholyl in water was employed. Thus the results with these two solvents were similar within the limits of the experimental method.

That blood pressure effects might be a result of extravasation of mecholyl into perisynovial tissues, and absorption thence, was ruled out by the deliberate injection of varying doses of the lrug into the infra-patellar fat pads of both joints of four cats. After two of these injections there was a blood pressure effect of the intravenous type -immediate and sharp-bearing no resemblance to the type of tracing obtained with intra-articular injection. Evidently in these two instances some of the mecholyl had entered a small vein. Following the injection of the fat parls of the remaining six joints, with amounts of mecholyl similar to those used in the regular intra-articular injections, no effect on the blood pressure was obtained.

The possibility of a local effect of mecholy in promoting absorption of itself from joints had to be considered. Whatever local vasodilator effect this drug might have was certainly very slight, however. That the observed rapidity of absorption from joints of an aqueous solution of a drug might have been due solely to local vasodilatation was ruled out by the rapicl absorption of other drugs than mecholyl (pituitrin and pilocarpine) described below.

Since the enzyme choline-esterase was known to be present in various body fluids, it was obvious that some of the loss of mecholyl from joints might have been due to the activity of this enzyme in the synovial fluicl. To test this. the synovial fluid from each of five cat knee joints was incubated in vitro with various doses of mecholyl. No significant destruction of mecholyl was obtained within a longer time than the 
drug remained in the joints in any of the experiments.

Since only very small amounts of synovial fluid could be obtained from the cat, entailing considerable dilution when mecholyl was added, similar tests were carried out with cow synovial fluid. Cow serum and synovial fluid were collected at the abbatoir under oil, brought to the laboratory in ice, and at once centrifuged. Dilutions of mecholyl, corresponding to the dilutions used in the absorption experiments, were then made up both in serum and synovial fluid, were divided into samples, and were placed in the incubator at $37^{\circ}$ C. The different samples were removed after varying intervals of time and tested on the blood pressure of a cat for their mecholyl content, with the following results.

With serum there was considerable loss of potency in 35 minutes; marked loss in 55 minutes; nine-tenths loss in 1 hour 8 minutes; complete loss in 1 hour 50 minutes. With synovial fluid no loss of potency was detected in 1 hour 50 minutes in a sample of clear fluid, although a somewhat blood-tinged sample showed a slight loss at 1 hour 8 minutes. There was two-fifths to three-fifths loss of potency in $2 \frac{1}{2}$ hours, and only three-fifths loss, even in the blood-tinged sample, up to 4 hours.

The above data supported the belief that within the time limits of the experiments herein reported, loss of mecholyl by enzymatic destruction within the joint was not a complicating factor. An incidental finding, however, was that both cat and cow synovial fluid apparently contained a small amount of choline-esterase.

As a final test of whether or not this enzyme constituted a source of error, any choline-esterase which might have been present in a cat joint was inactivated by injecting into the joint eserine (3) along with the mecholyl, and then performing a standard absorption experiment. The opposite joint was injected with mecholyl alone. Upon the final washing out of the joints, a somewhat greater recovery of mecholyl was obtained from the non-eserinized joint, indicating at least that within the limits of the experimental method, eserinization did not cause less loss of cholinecompound.

Experiments on lymphatic absorption of mecholyl from joints (to be described below) necessitated an estimation of the choline-esterase content of cat lymph, in order that any mecholyl which might gain access to the lymph from a joint could be adequately protected with eserine while its potency was being tested. For the purpose of this study, mecholyl was incubated at $37^{\circ}$ C. with cat serum and lymph, with the following results.

With serum, mecholyl retained some of its potency for 20 minutes, but lost all of it after 40 minutes. With lymph, collected from the thoracic ducts of 5 normal cats, after incubation for 15 and 30 minutes, or after standing at room temperature for 30 minutes, there was marked destruction of mecholyl; after incubation for 45 and 60 minutes there was complete destruction.

Thus the control data showed mecholyl to be adequately stable for use in an investigation of this type; they demonstrated that the drug retained its potency throughout the course of the experiments; and they substantiated the sufficient accuracy of the various experimental techniques employed.

\section{EXPERIMENTS WITH ADRENALIN}

Additional studies in which adrenalin was used in conjunction with mecholyl gave further insight into the mechanism of absorption of the latter drug from joints. In these experiments a large dose of mecholyl ( $0.025 \mathrm{mgm}$.) was injected into 5 joints (of 3 cats), producing a marked and sustained fall of blood pressure (Figure 4, upper tracing). While this fall was still in progress, the injection into 4 of these 5 joints of adrenalin 0.1 cc. of $1: 1000$ led to a prompt rise of the blood pressure to normal (Figure 4, lower tracing). This inhibiting effect of adrenalin persisted for at least 2 hours. Furthermore, even a small dose 'of adrenalin (0.1 cc. of $1: 10,000)$ prevented the large dose of mecholyl from causing a subsequent fall of blood pressure.

The remaining joint of the third cat in this series was injected with adrenalin initially, producing no significant effect on the blood pressure (as had been observed regularly in preliminary studies). The same dose of mecholyl used above, injected subsequently into this joint, led to no drop in blood pressure, but produced instead a moderate gradual rise. This rise with mecholyl following adrenalin was confirmed on several other occasions, but no experimental attempt was made to analyze it. It might suggest that mecholyl, having diffused into the subsynovial tissues, 




83

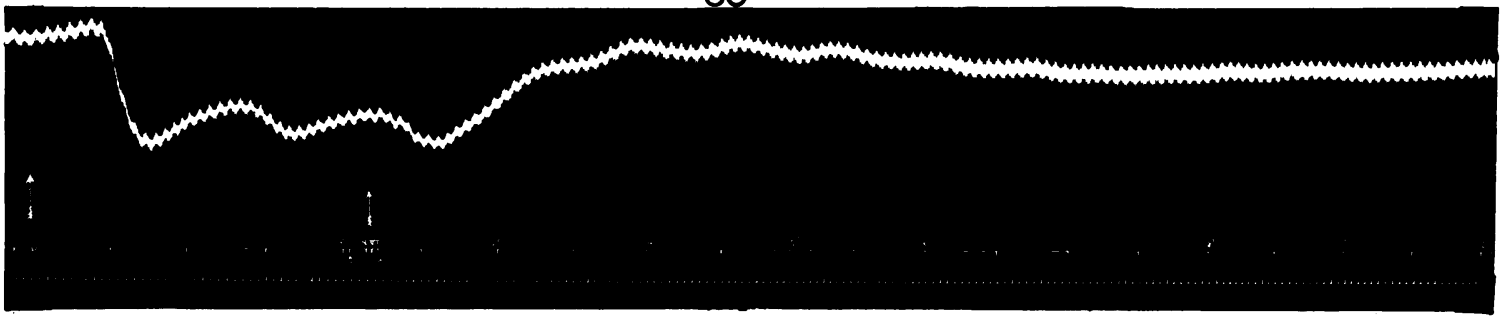

Fig. 4. Cat 83-Mecholyl (0.025 igir.) Injected into Each Joint (First Arrows)

Both exercised. Upper tracing, for one joint, shows blood pressure effect of this large dose over a period of 10 minutes. Lower tracing, for the other joint, shows rapid recovery of blood pressure to normal after injection of adrenalin, $0.1 \mathrm{mgm}$. (second arrow) into the same joint $2 \frac{1}{4}$ minutes after the mecholyl injection.

permitted by local action on the capillaries some of the adrenalin to be absorbed.

The adrenalin experiments indicate that this drug, injected after mecholyl, blocks absorption of the latter, presumably by constricting the subsynovial capillaries. Thus these experiments point to another important general conclusion, confirming and emphasizing the previous impression based upon the great rapidity with which mecholyl was absorbed from joints, that,

6. A solution of mecholyl is absorbed from joints in large part by way of the blood capillaries.
EXPERIMENTS WITH PITLITRIN AND PILOCARPINE

The absorption of pituitrin and pilocarpine from the knee joint was also studied. Two cats which had already been used in mecholyl experiments gave evidence that pituitrin was readily absorbed. A full experiment on another cat demonstrated a striking absorption of pituitrin, apparently somewhat more rapicl with exercise than without. In each instance the blood pressure rose gradually, accompanied by an increasing pulse swing, reaching a maximum in about 10 minutes (Figure 5). It will be noted that the

\section{5}

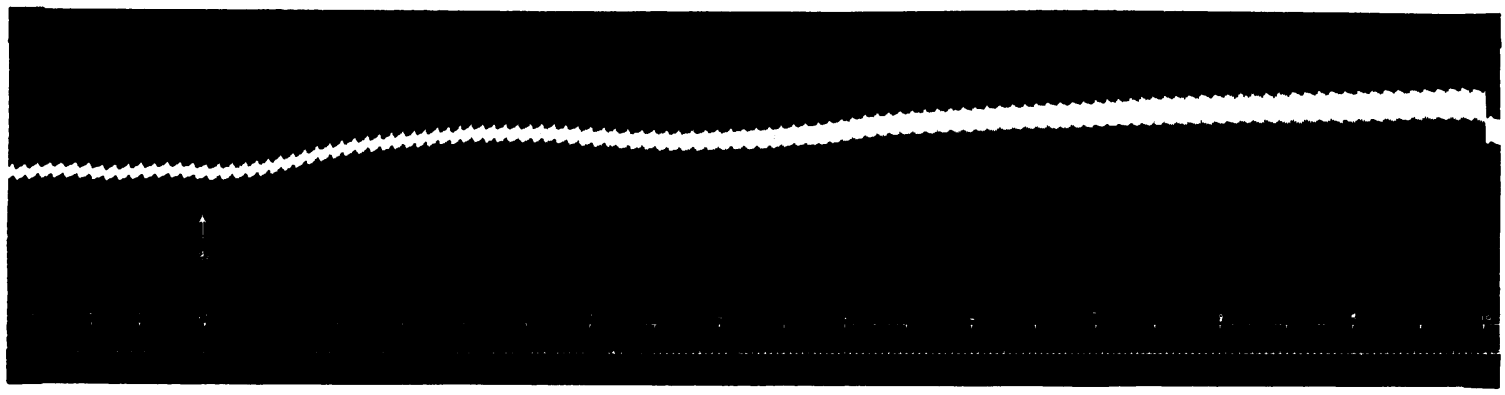

Fig. 5. Cat 85-Pitritrix (Slrgical) 0.25 co. Injectei) into Joint Exercised for 10 minutes. Tracing shows gradual rise of blood pressure. 
effect on the blood pressure was apparent in less than 30 seconds after the intra-articular injection.

Pilocarpine was injected into one knee joint of a cat which had previously been injected with mecholyl, the latter having been washed out carefully. A strongly hypertonic solution of pilocarpine was used, $23 \mathrm{mgm}$. in $0.4 \mathrm{cc}$. distilled water. The joint was exercised. No tracing to demonstrate the rapidity of early absorption was made in this experiment, but about 2 minutes after the injection, the cat began to exhibit toxic symptoms. These soon became so marked that the cat was sacrificed. The joint was then found to contain a large effusion which had not been present before the injection. Thus the hypertonic solution of pilocarpine had drawn fluid into the joint while at the same time the drug was being absorbed. This interchange must have been via the blood capillaries, for one could scarcely expect sufficient pilocarpine to pass up the femoral lymphatics and thoracic duct within two minutes' time to produce systemic toxicity.

A significant point is thus brought out by these experiments with other drugs, confirming the results with mecholyl, that,

7. Aqueous solutions of pituitrin and pilocarpine are readily absorbed from joints, apparently via the blood stream.

\section{LYMPHATIC ABSORPTION}

The preceding experiments suggested that various drugs in aqueous solution were absorbed from joints at least in considerable proportion via the blood capillaries. A few experiments were carried out in an attempt to ascertain whether any large amount of the mecholyl absorbed from the knee joint was conveyed by way of the lymphatics. In these experiments mecholyl, in the usual intraarticular dose of $0.003 \mathrm{mgm}$., was injected into the knee joints of cats of which the thoracic duct had been cannulized ${ }^{\circ}$ (all communications between lymphatic and vascular systems having been ligated). These cats were eserinized to prevent enzymatic destruction of choline-compound in the lymph. Preliminary studies had indicated that $1 \mathrm{mgm}$. of eserine per kilogram was sufficient for

- We are indebted to Dr. Madeleine Field Warren of the Department of Physiology for performing this operation for us. this purpose. This was injected intravenously, with the addition of atropine $0.43 \mathrm{mgm}$. to prevent too marked a fall of blood pressure (3). These drugs had to be reinjected in smaller doses from time to time during an experiment, as incubation of lymph samples with mecholyl indicated that the eserinization was wearing off. After intra-articular injection of a cat, samples of thoracic-duct lymph were collected for 15minute periods up to $11 / 2$ to 2 hours, and each sample was given intravenously to another cat prepared in the routine manner for recording the effects on blood pressure.

Nine intra-articular injections were carried out on 4 cats from which lymph samples were collected. Following only 2 of these 9 injections was a detectable amount of vasodepressor substance present in any 15-minute lymph sample. One of these instances was with the 30 to 45minute sample in the experiment with the exercised joint of a well eserinized cat of which the abdomen was vigorously massaged to promote the flow of lymph. The drop in blood pressure in the testing cat caused by this sample was 43 $\mathrm{mm}$. $\mathrm{Hg}$. The other instance was with a 45 to 60-minute sample from a cat which was not fully eserinized. The mecholyl-like effect produced on the blood pressure of the testing cat by this sample was very slight, and therefore somewhat indefinite. Furthermore, this sample was obtained in the experiment with the resting joint of the cat, whereas from the exercised joint of the same cat no trace of lymphatic absorption was detected.

Following 7 of the 9 intra-articular injections, both with exercise and rest, no vasodepressor substance could be found in any of the lymph samples. One of the cats in this negative group appeared not to have been fully eserinized. In the final experiment, the lymph flowed freely without abdominal massage, making it exactly comparable to the experiments described in the previous portions of this paper. When no absorption of mecholyl into the lymph could be detected from either the resting or the exercised joint, the drug was again injected into each joint, and both were exercised in conjunction with massage of the abdomen. Tests indicated that the cat had remained eserinized throughout. Thus, even in this ideal experiment, the presence of mecholyl in the thoracic duct could not be demonstrated. 
It is of course possible that some mecholyl may have diffused out of the lymph channels before reaching the upper end of the thoracic duct, or that the concentration of mecholyl in the lymph samples was too small to have been detected regularly by the method used. The limit of sensitivity of several of the testing cats was found to vary between 0.00005 and $0.000075 \mathrm{mgm}$. of mecholyl, which is much inferior to the sensitivity of leech muscle preparations for the detection of acetylcholine (3). However, the cat blood pressure method, already being employed in this investigation, appeared sufficiently accurate to permit in a brief additional study the demonstration of whether a large proportion of the drug absorbed from the joint was in fact borne by the lymphatics. This additional study has indicated that some mecholyl may reach the upper end of the thoracic duct from the joint after 30 minutes, but not in detectable quantity before this time. Hence the experiments on lymphatic absorption support also the main thesis of this discussion, that,

8. Mecholyl is absorbed from joints primarily by way of the subsynovial blood capillaries.

\section{DISCUSSION}

It has been well established that certain proteins (1) and dyes of colloidal dimensions (4, 5 , $6,7)$ leave joints chiefly or entirely by way of the subsynovial lymphatics. The experiments with mecholyl and other drugs herein described suggest that substances of small molecular dimensions leave joints by way of the blood stream. The experiment with slow intravenous infusion of mecholyl demonstrated that it was possible for all the drug absorbed from the joint to have entered the blood stream directly. Undoubtedly materials in aqueous solution leave joints in part also via the subsynovial lymphatics, as demonstrated in two instances above, and as concluded by certain workers $(7,8)$ with dyes, but they do so much more slowly. Presumably the bloodvascular absorption of aqueous solutions is the more important avenue of exit in the acute physiological and pathological processes of joints.

The increased absorption of compounds in aqueous solution, such as mecholyl, which was observed with passive exercise would be an expected sequel to the local pressure changes (9) and the increased local blood flow which would be brought about by rhythmic flexion and extension of a joint. The further increased absorption of such a solution from acutely inflamed joints would also be a natural accompaniment of the further increased local vascularity. There is probably an exchange of water and various substances between the blood of the capillaries and the synovial fluid of the joint cavity. In the normal joint this exchange results in a well balanced equilibrium. It is reasonable to suppose that the enhanced absorption of aqueous solutions, observed during a physiological condition such as mild exercise, is exactly balanced by an enhanced effusion of fluid. Likewise, it must be a fact that the greatly promoted absorption of mecholyl under certain pathological conditions, such as was shown with acutely inflamed joints, is accompanied by a similarly great promotion of aqueous effusion. When the pathological state attains a certain character, effusion exceeds absorption, and hydrops of the joint occurs. The exact character of this pathological state in physicochemical terms is as yet undetermined. However, an equivalent condition was produced experimentally in the joint from which a hypertonic solution of pilocarpine was being absorbed while a marked effusion was in progress. Various observers have reported that in human joint effusions the protein content of the synovial fluid is higher than the normal $(10,11,12,13)$. This increased protein content should induce an intra-articular osmotic state comparable with the hypertonic solution of the above experiment. It is in the rapid alterations in volume and electrolyte character of synovial fluid, attendant upon acute osmotic variations, that interchange of materials in aqueous solution between joint cavity and blood capillaries evidently plays a major rôle.

\section{SUMMARY AND CONCLUSIONS}

1. Experiments, devised to study the manner of absorption of mecholyl in aqueous solution from normal and certain pathological joints of cats, under conditions of rest and exercise, are described.

2. Solutions of mecholyl and other drugs were promptly transferred from resting normal joints to the blood stream. 
3. Passive exercise hastened and increased this absorption.

4. Acute inflammation enhanced absorption both from resting and exercised joints, severe inflammation more markedly than mild inflammation.

5. A certain proportion of the mecholyl absorbed from the joints appeared to be removed slowly by way of the subsynovial lymphatics.

6 . The greater and most rapidly absorbed proportion of the drug was removed by the blood capillaries.

7. This blood-vascular interchange is interpreted as being of primary importance in the acute changes in the volume and in the electrolytes of synovial fluid.

\section{BIBLIOGRAPHY}

1. Bauer, W., Short, C. L., and Bennett, G. A., The manner of removal of proteins from normal joints. J. Exper. Med., 1933, 57, 419.

2. Unpublished data.

3. Dale, H. H., and Feldberg, W., The chemical transmitter of vagus effects to the stomach. J. Physiol., 1934, 81, 320.
4. Kuhns, J. G., Lymphatic drainage of joints. Arch. Surg., 1933, 27, 345.

5. Key, J. A., The mechanisms involved in the removal of colloidal and particulate carbon from joint cavities. J. Bone and Joint Surg., 1926, 24, 666.

6. Sigurdson, L. A., The structure and function of articular synovial membranes. J. Bone and Joint Surg., 1930, 12, 603.

7. Rynearson, E. H., Studies on the physiology of joints. Proc. Staff Meet., Mayo Clinic, 1928, 3, 171.

The macrophage in absorption from the synovial cavity. J. Bone and Joint Surg., 1931, 13, 127.

8. Allen, A. L., Penetrating wounds of the knee. I. The production of experimental arthritis. South African J. M. Sc., 1935, 1, 31.

9. Smith, M., and Campbell, J. R., Jr., Observations on the lymphatic drainage of joint cavities. Proc. Soc. Exper. Biol. and Med., 1929, 26, 395.

10. Forkner, C. E., The synovial fluid in health and disease, with special reference to arthritis. J. Lab. and Clin. Med., 1930, 15, 1187.

11. Allison, N., Fremont-Smith, F., Dailey, M. E., and Kennard, M. A., Comparative studies between synovial fluid and plasma. J. Bone and Joint Surg., 1926, 8, 758.

12. Collins, D. H., The pathology of synovial effusions. J. Path. and Bact., 1936, 42, 113.

13. Unpublished data. 